\title{
Histopathological Study of Psoriasis and Its Grading According to Trozak Scoring System
}

\author{
Neelu Vashist ${ }^{1}$, Ila Sharma ${ }^{1 *}$ and Mukul Sharma ${ }^{2}$ \\ 'Department of Pathology, Government Medical College, Kota, Raj. India \\ ${ }^{2}$ Department of Dermatology and Veneral Diseases, Government Medical College, Kota, Raj. India
}

\section{ABSTRACT}

Background: Psoriasisis a chronic papulosquamous disorder of undefined etiology, occuring in 0.09 to $11.4 \%$ population. Different clinical types occur with varying histopathology. Early and Late onset psoriasis have been defined based on age at presentation.

Methods: Cases diagnosed as Psoriasis, both clinically and histologically, were examined for the individual features of Psoriasis. Histomorphological grading of the lesions was also done using the Trozak scoring system.

Result: The mean age of the patients was 42.26 with a male predominance (M:F=3.5: 1). Early onset cases constituted $71 \%$ of the total cases, while late onset cases were $29 \%$. The histopathological features were found to be variably present. Acanthosis was observed in $96 \%$ cases. Hyperkeratosis $(94 \%)$, parakeratosis $(66 \%)$, club shaped rete ridges $(88 \%)$ with regular elongation of rete ridges $(72 \%)$ with, suprapapillary plate thinning (74\%), hypogranulosis $(72 \%)$, elongation and edema of dermal papillae $(88 \%)$ were other features seen in majority of cases. Perivascular mononuclear infiltrate in upper dermis of papillae (76\%), dermal (76\%) and epidermal (78\%) neutrophils, spongiform pustules of Kogoj (24\%), Munro's microabscess (28\%), were the inflammatory changes observed, present to a greater extent in the early onset cases than the late onset cases. The Trozak scoring system gave an average score of 9.44, with no significant difference in the early and late onset cases of Psoriasis.

Conclusion: Entirely typical histological features are found only in a small percentage of biopsy specimens, even if only clinically typical lesions of psoriasis are examined.

Keywords: Psoriasis, Histology, Skin Disease, Trozak Grading

\section{Introduction}

Psoriasis (Greek. Psora, the itch) ${ }^{[1]}$ is a chronic, relapsing papulosquamous disorder of undefined etiology, characterized by localized or generalized, mostly symmetrical, sharply demarcated plaques and papules, usually covered with white or silver scales ${ }^{[2]}$ on the scalp, elbows, knees, lumbosacral area and in the body folds. Prevalence of psoriasis in countries vary between $0.09 \%{ }^{[3]}$ and $11.4 \%{ }^{[4]}$ Psoriasis can occur at any age, and is most common in the age group 50-69. ${ }^{[5]} \mathrm{It}$ is considered equally prevalent in both sexes. ${ }^{[2,6]}$ A seasonal variation of the disease, with a higher frequency of flares in winter and spring, has been seen. ${ }^{[7]}$ The prevalence of Psoriasis varies among different ethnic groups and geographical locations, more common in colder northern zone compared to tropical zone.

Clinical Presentations: Psoriasis affects skin and nails, along with associated arthritis and systemic diseases. The most frequently reported symptoms are scaling of the skin(92\%), itching, erythema, fatigue, swelling, burning and bleeding.$^{[8]}$ Scale is characteristically silvery white and can vary in thickness. Removal of scale may reveal tiny bleeding points (Auspitz sign). The common clinical types of Psoriasis are summarised in the International Psoriasis Council classification and consist of plaque psoriasis, guttate psoriasis, pustular psoriasis, erythrodermic psoriasis, eczematous psoriasis, and photosensitive psoriasis. ${ }^{[9]}$ Plaque type Psoriasis, also known as Psoriasis vulgaris, is the commonest form representing 70-80\% of psoriatic patients. ${ }^{[10]}$ Histology also varies among the different clinical variants. An identification of two subtypes of Psoriasis based on the "age of disease onset" has been described by Henseler and Christophers; Type I or Early Onset Psoriasis, presents at $\leq 40$ years of age and Type II or Late Onset Psoriasis, developing after the age of 40. Multiple studies have shown that these two subtypes are clinically, genetically and immunologically heterogeneous. Early onset psoriasis, in contrast to Late onset psoriasis, is probably hereditary and shows HLA-Cw6 positivity and parental history with an irregular course, stronger tendency to become generalized, ${ }^{[7]}$ more nail involvement and greater psychosocial impact. ${ }^{[1]}$ Differential diagnoses of Psoriasis include Allergic contact dermatitis, Bowen's disease, Lichen planus, Lichen simplex chronicus, Mycosis fungoides, Parapsoriasis, Nummular dermatitis, Onychomycosis, Pityriasis alba, Pityriasis rosea, Pustular eruptions, Seborrheic dermatitis, Syphilis and Tinea. ${ }^{[12]}$ 
Histology of Psoriasis: In psoriasis there is rapid turnover of the epidermis due to accelerated proliferation and differentiation of epidermal keratinocytes. All phases of the cell cycle are shortened to such an extent that the total cell cycle lasts only 1.5 days insteal of 30 days. H\&E stained histological sections of Psoriasis show a characteristic psoriasiform pattern. There is abnormal hyperproliferation and differentiation of keratinocytes, resulting in diffuse hyperplasia (acanthosis) of the Malpighian layer (stratum basale and stratum spinosum), with elongation of the rete ridges and presence of nucleated keratinocytes in the top layer of the epidermis (parakeratosis). Absence of the granular layer (hypogranulosis) and suprapapillary plate thinning have been found to be statistically significant contributors to clinicohistological concordance in the diagnosis of psoriasis. Increased mitosis in the basal layer as well as in suprabasal layers is prominent. ${ }^{[13]}$ The suprabasal mitotic index was used in a recent study as a diagnostic criteria for psoriasis. ${ }^{[14]}$ There is epidermal spongiosis and papillary dermal elongation and edema. A series of abnormal events take place in the dermal microvasculature early on. These include increased endothelial cell proliferation and neovascularisation resulting in the dermal papillary vessels appearing dilated, elongated and tortuous, with increased blood flow and permeability. Inflammatory infiltration appears to be the central reason for the entire pathogenesis of Psoriasis. The most characterstic finding is the presence of neutrophilic collections in the stratum corneum (Munro's microabscesses) and/or the subcorneal area (Kogoj's spongiform pustules). It is thought that neutrophils are recruited by the neutrophil-attracting chemokine interleukin-8 (CXCL8) synthesized by the CD4 T cells. These neutrophils then migrate through the epidermis. A dense lymphocytic infiltrate is present in the dermis. Occasionally, localised or diffuse eosinophilic infiltrate or mast cells are present in the dermis. In his study on late onset Psoriasis, Theodorakopoulou ${ }^{[7]}$ noted statistically significant differences in inflammatory components between EOP and LOP, with LOP showing a more intense inflammatory infiltration and a high infiltration of $\mathrm{CD} 4+$ and $\mathrm{CD} 3+$ cells in the epidermis of clinically involved skin sections, irrespective of gender or disease duration. According to De Rosa, psoriasis is a dynamic dermatosis that changes during the evolution of an individual lesion; we can classify it into early stage, advanced stage, and late lesions. ${ }^{[15]}$ At different stages of Psoriasis, the histological manifestations are present in varying degrees. ${ }^{[16]}$

Grading of Psoriasis: Dermatologists focus on clinical severity assessment tools in diagnosing Psoriasis, including the Psoriasis area and severity index (PASI) score, which have innate limitations as sole indicators. The Trozak scoring system is a histological tool for quantifying features of Psoriasis. ${ }^{[17]}$ The advantage of this method is that it provides an objective and measurable grade to the histopathological biopsy of psoriatic lesion. The disadvantage lies in that the clinicopathological correlation has not yet been demonstrated strongly.

In this study, we intend to observe the characteristic histopathological features of Psoriasis and further grade them by the Trozak Scoring System to define the severity of Psoriasis and its possible association with early and late onset.

\section{Materials and Methods}

The present study included patients at Government Medical College, Kota (Rajasthan), from July 2014 to June 2016. Patients in whom Psoriasis was clinically suspected, underwent biopsy from the leion site. After processing, sections were stained with Haematoxylin and Eosin and subjected to microscopy under low power and high-power lens. The diagnosis of Psoriasis was confirmed histologically. The cases which were diagnosed as Psoriasis, both clinically and histologically, were then examined for the individual features of Psoriasis. Morphological grading of the lesions was also done by the Trozak scoring system. The scoring is done based on the following features: Parakeratosis and hypogranulosis each given a score of 0-Absent 1-Focal 2-Diffuse; regular elongation of rete ridges and perivascular mononuclear infiltrate in the papillary dermis each given a score of 0-Absent 1-Present; suprabasal mitoses, "club-shaped" rete ridges, suprapapillary plate thinning and oedematous dermal papillae each given a score of 0-Absent 2-Present; Munro's microabscesses and Kogoj pustules each given a score of 0-Absent 3-Present; A final total score of the above features is given from $0-19 .{ }^{[7,17]}$

\section{Result}

Among the 50 cases, the age of patients ranged from 9 to 70 years. 12 patients lied in 41-50 years age group. (Figure 1) The average age was 42.26 .

Early onset Psoriasis was found in $29 \%$ and Late onset Psoriasis was seen in $71 \%$ of the cases. The mean age among Early onset cases was 23.76 and among Late onset cases was 55.66.

Male patients accounted for $78 \%$ of the biopsies while $22 \%$ were females. The male:female ratio was $3.5: 1$. When assessed in relation to early and late onset Psoriasis, the females had more proportion of early onset cases while the males had more of late onset cases. (Figure 2) 
The findings of individual histological features were as tabulated in Table 1. Inflammatory infiltration was also assessed separately in terms of early and late onset cases which gave results as shown in Table 2. (Figures 3 to 6)

The Trozak score was calculated by adding the scores of all the points included in the scoring system. Mean Trozak score obtained was 9.44 for all 50 cases. The score for EOP (9.48) was only slightly higher than for LOP (9.41). The highest average score was obtained in 30-40 years age group (12.75).

\section{Discussion}

Of the 50 patients in our study, the age ranged from 9 year in one case to maximum of 70 years. Most number of patients were found to lie between 41 to 50 years of age. The mean age was found to be 42.26 . The age related findings were similar to the results of a population based study by Murat

Table 1: Histological Features of Psoriasis Observed.

\begin{tabular}{|c|c|c|c|}
\hline Histologic feature & Absent & \multicolumn{2}{|c|}{ Present } \\
\hline Acanthosis & $2(4 \%)$ & \multicolumn{2}{|c|}{$48(96 \%)$} \\
\hline hyperkeratosis & $3(6 \%)$ & \multicolumn{2}{|c|}{$47(94 \%)$} \\
\hline Club shaped rete ridges & $6(12 \%)$ & \multicolumn{2}{|c|}{$44(88 \%)$} \\
\hline Elongation and edema of dermal papillae & $6(12 \%)$ & \multicolumn{2}{|c|}{$44(88 \%)$} \\
\hline Dermal lymphocytes & $6(12 \%)$ & \multicolumn{2}{|c|}{$44(88 \%)$} \\
\hline Epidermal neutrophils & $11(22 \%)$ & \multicolumn{2}{|c|}{$39(78 \%)$} \\
\hline Perivascular mononuclear infiltrate in upper dermis of papillae & $12(24 \%)$ & \multicolumn{2}{|c|}{$38(76 \%)$} \\
\hline Prominent dermal capillaries & $12(24 \%)$ & \multicolumn{2}{|c|}{$38(76 \%)$} \\
\hline Dermal neutrophils & $12(24 \%)$ & \multicolumn{2}{|c|}{$38(76 \%)$} \\
\hline Suprapapillary plate thinning & $13(26 \%)$ & \multicolumn{2}{|c|}{$37(74 \%)$} \\
\hline Regular elongation of rete ridges & $14(28 \%)$ & \multicolumn{2}{|c|}{$36(72 \%)$} \\
\hline Spongiosis & $14(28 \%)$ & \multicolumn{2}{|c|}{$36(72 \%)$} \\
\hline Absent granular layer & $14(28 \%)$ & focal-27 (54\%) & diffuse-9 (18\%) \\
\hline Parakeratosis & $17(34 \%)$ & focal-23 (26\%) & diffuse-10 (20\%) \\
\hline Munro micro abscesses & $36(72 \%)$ & \multicolumn{2}{|c|}{$14(28 \%)$} \\
\hline Mitosis above basal cell layer & $37(74 \%)$ & \multicolumn{2}{|c|}{$13(26 \%)$} \\
\hline Spongiform pustule & $38(76 \%)$ & \multicolumn{2}{|c|}{$12(24 \%)$} \\
\hline
\end{tabular}

(Sorted according to decreasing presence)

Table 2: INFLAMMATORY CHANGES OBSERVED IN EOP AND LOP CASES.

\begin{tabular}{|l|c|c|}
\hline Inflammatory changes & EOP & LOP \\
\hline Munro's micro abscesses & $33.30 \%$ & $24.10 \%$ \\
\hline Spongiform pustule & $23.80 \%$ & $24.10 \%$ \\
\hline Epidermal neutrophils & $85.70 \%$ & $72.40 \%$ \\
\hline Dermal lymphocytes & $100 \%$ & $79 \%$ \\
\hline Dermal neutrophils & $95.20 \%$ & $62 \%$ \\
\hline Perivascular mononuclear infiltrate in upper dermis of papillae & $80.90 \%$ & $72.40 \%$ \\
\hline
\end{tabular}

(Inflammatory findings in epidermis in grey, dermis in white) 


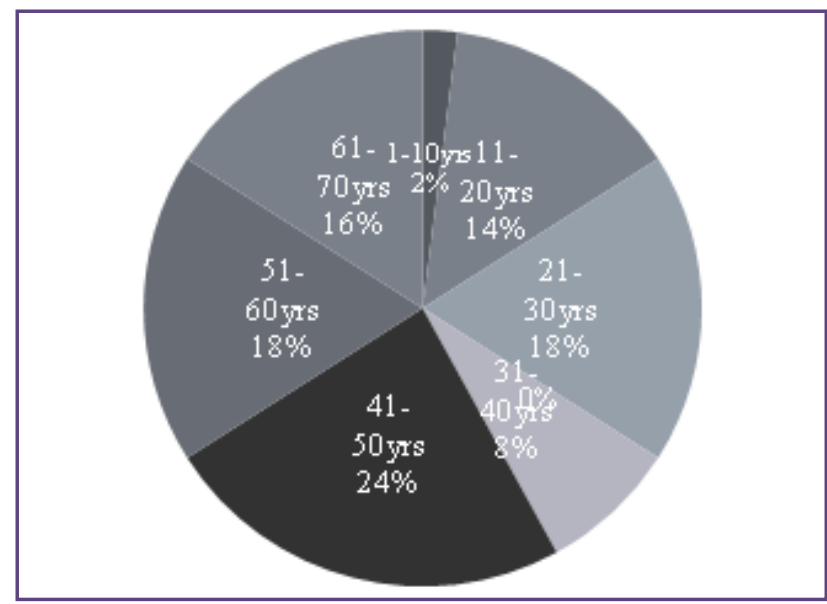

Fig. 1: Age wise distribution of cases of psoriasis.

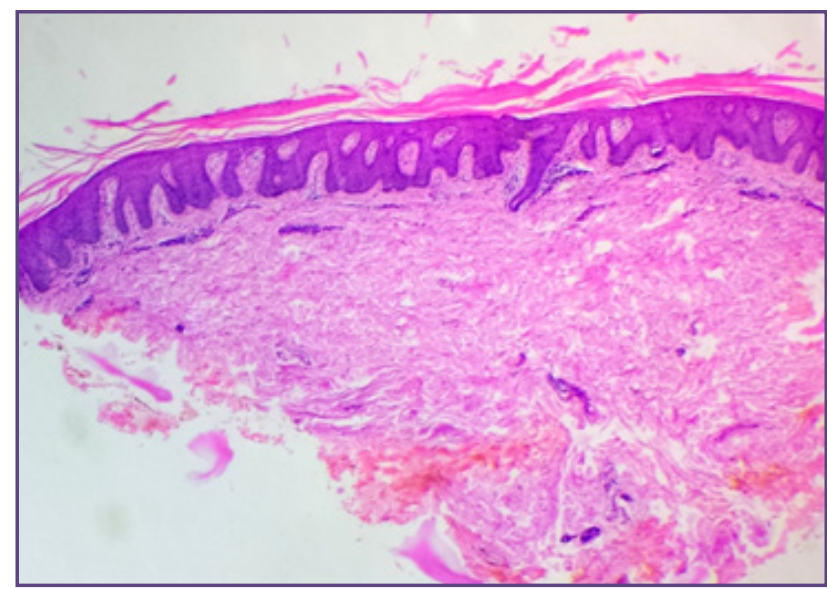

Fig. 3: Section shows hyperkeratosis, regular elongation of rete ridges with club shaped rete pegs and suprapapillary thinning. (H\&E,40x).

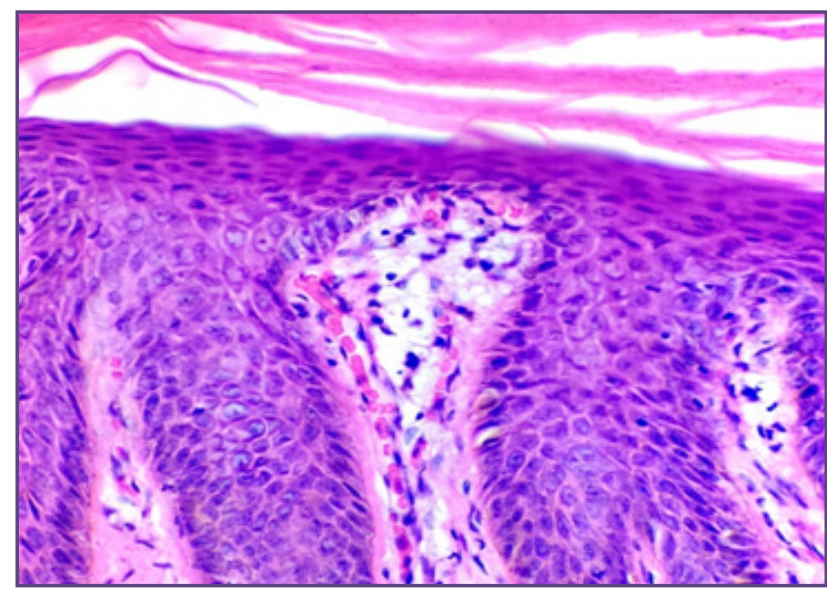

Fig. 5: Section shows thinning of the suprapapillary epidermal region, dilated capillaries in the papillary dermis with mononuclear infiltrate. (H\&E,400x).

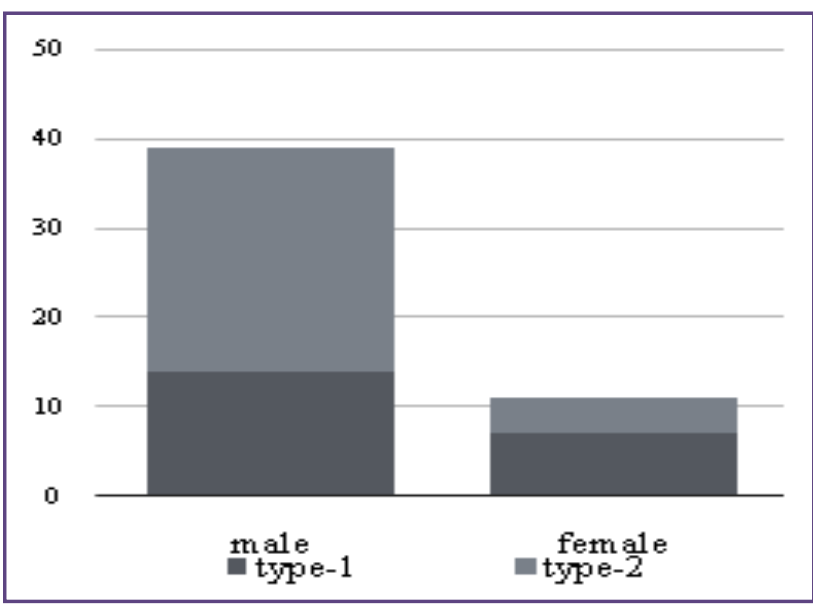

Fig. 2: Gender wise distribution of cases, divided further into Type-1(age<40years) and Type-2(age>40years).

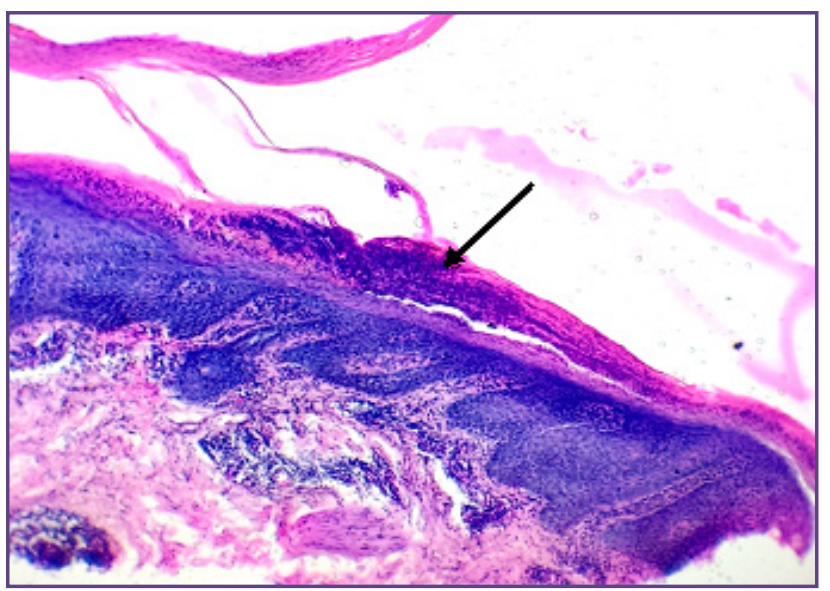

Fig. 4: Section shows hyperkeratosis, parakeratosis, Micromunro's abscess (arrow), acanthosis with elongation of rete ridges and suprapapillary thinning. (H\&E,100x).

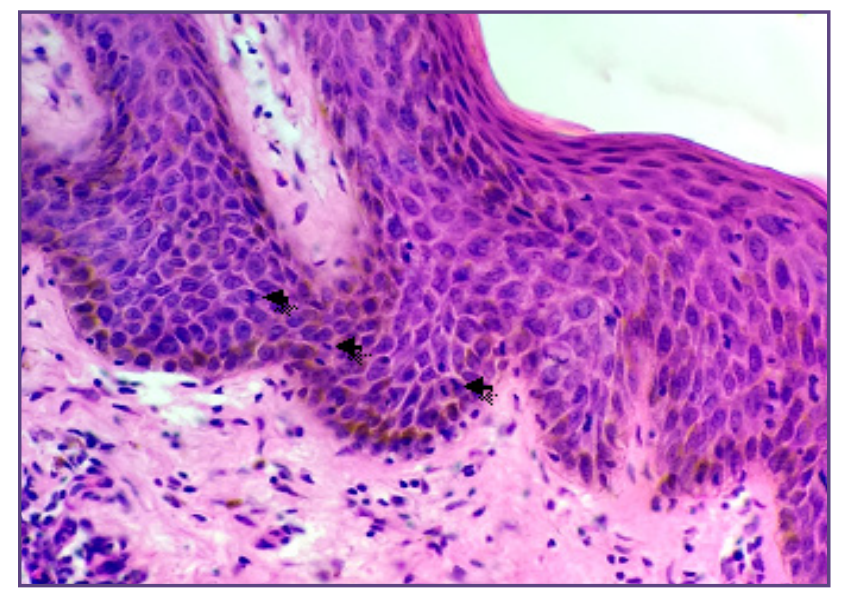

Fig. 6: Section shows presence of mitoses in the suprabasal region of epidermis(arrowheads), epidermal neutrophils and chiefly mononuclear infiltrate in the dermis, occasional eosinophil also visible. (H\&E,400x). 
Icen et $\mathrm{al}^{[18]}$ where the mean age at diagnosis was 43.2 . Also, in a study by Moorchung et al, ${ }^{[19]} 88$ cases were analysed, in which the mean age came to be 38.9 years with a range of 13 to 76 years.

On categorization of our cases according to Henseler and Christophers, ${ }^{[20]}$ late onset Psoriasis was more common (71\% cases) with mean age of 55.66 years. Early onset Psoriasis (29\% cases) had mean age of 23.76 years at presentation. In comparison, another asian study by Ejaz et $\mathrm{al}^{[21]}$ noted $51.1 \%$ early onset cases with peak age of 19.67 years and $49.9 \%$ late onset cases with peak at 41.85 years.

The incidence of psoriasis in adult men and women is usually reported to be about equal. ${ }^{[16]}$ However, some studies indicated that psoriasis is more common in men.

${ }^{[2]}$ Male to female ratio of psoriasis in different studies from India by Bedi, ${ }^{[22]}$ Kaur et al, ${ }^{[23]}$ Raghuveer et $\mathrm{al}^{[16]}$ varied from 2 to $4: 1$. The lower incidence observed in females from these parts of world may be due to their being less attentive to health, and occurrence of psoriasis over covered parts. In the present study, there was a male preponderance, with male to female ratio of $3.5: 1$, similar to the above studies.

The results obtained on histopathological study when compared with similar features included in other studies showed certain corresponding data along with contrasting findings in case of some features. This can be explained on the basis of varying degrees of activity of the disease. Acanthosis as well as hyperkeratosis and parakeratosis are result of proliferation and decreased turnover time that occurs in Psoriasis ${ }^{[19]}$ and are hence commonly found. In our study, the feature present in maximum proportion of cases was acanthosis, which was observed in $96 \%$ cases. The results were comparable with findings of study by Puri et $\mathrm{al}^{[24]}$ who reported it in $100 \%$ cases of Psoriasis vulgaris, and Aydin et $\mathrm{al}^{[25]}(88.20 \%)$. In our study, occurance of hyperkeratosis ( $94 \%$ cases) was comparable to results of study by Raghuveer et $\mathrm{al}^{[16]}(89 \%)$. Parakeratosis was present diffusely in $20 \%$ of the cases, while it was focal in $46 \%$. This was similar to the findings of Raghuveer et $\mathrm{al}^{[16]}$ who observed confluent parakeratosis in $31 \%$ cases and focal parakeratosis in $46 \%$ cases.

Club shaped elongation of rete ridges or psoriasiform hyperplasia is considered one of the defining features of Psoriasis. Club shaped rete pegs were seen in $88 \%$ cases in our study while Park et $\mathrm{a}^{[26]}$ noted clubbing in $100 \%$ cases. There was regular elongation of rete ridges in $72 \%$ cases, which corresponded with the findings of Raghuveer et al ${ }^{[16]}$ $(75 \%)$ and Puri et $\mathrm{al}^{[24]}(81.25 \%)$. Kim et $\mathrm{al}^{[27]}$ got higher numbers with $93 \%$ of the cases showing regular elongation of rete ridges. Elongation and edema of dermal papillae was seen in $88 \%$ cases, which was similar to the findings of Kim et $\mathrm{al}^{[27]}$ who found $93 \%$ of cases showing this feature in their study.

Absence of granular layer, either focal or diffuse, is another characterstic feature of Psoriasis. In the present study, there was focal absence of granular layer in 54\% cases and in $18 \%$ cases diffuse agranulosis was seen. Our results corresponded to the results of Raghuveer et $\mathrm{al}^{[16]}$ (51\% and $19 \%$ respectively). Suprapapillary plate thinning was seen in $74 \%$ cases and corresponded with finding of Park at al ${ }^{[26]}$ (73.3\%). These features are responsible for Auspitz sign.

Micromunro's abscess and spongiform pustules of Kogoj are considered the most specific findings of psoriatic process. ${ }^{[19]}$ In our study, Micromunro's abscess and spongiform pustules of Kogoj were seen in $28 \%$ and $24 \%$ cases respectively. These were lower in comparison to the findings of Raghuveer et $\mathrm{al}^{[16]}(58 \%$ and $30 \%$ respectively). The reason may lie in delayed medical consultation by patient from time of presentation and some level of self medication.

We observed the presence of dermal inflammatory infiltrate in most of the cases of Psoriasis, with dermal lymphocytes seen in $88 \%$ cases which was comparable with results of Raghuveer et al ${ }^{[16]}(98 \%)$. Dermal neutrophilic infiltrate was seen in $76 \%$ cases, while Puri et al ${ }^{[24]}$ found it in $43.75 \%$. Occasionally, localised or diffuse eosinophilic infiltration of dermis is encountered. A prominent dermal eosinophilic infiltration in a background of psoriasiform dermatitis is considered a marker of drug-induced psoriasiform eruptions. Only a limited number of reports exist on the functional role of eosinophils in non-drug-induced psoriasis. ${ }^{[7,28]}$ One of the cases we studied showed presence of eosinophilic dermal infiltrate.

On differentiating the inflammatory changes in early and late onset cases, a higher proportion of cases in early onset group were seen to contain inflammatory changes in the dermis and epidermis, including Munro's microabscesses and spongiform pustules of Kogoj. These results are in contrast to the results of Theodorakopoulou, who found that the late onset cases were associated with more inflammatory changes, although there were no significant morphological differences between the two subtypes of psoriasis. ${ }^{[7]}$

Other findings including prominent, dilated blood capillaries and perivascular lymphocytic infiltrate were each observed in $76 \%$ of the cases in the present study. Aydin et a ${ }^{[25]}$ noted similar occurance of dilated capillaries (76.5\%). Puri et $\mathrm{al}^{[24]}$ in their study observed a lower but comparable value of PVLI (62.5\%). Spongiosis was seen 
in $72 \%$ cases in our study, while results ranged from $47.1 \%$ and $50 \%$ in studies by Puri et al ${ }^{[24]}$ and Aydin et al ${ }^{[25]}$ respectively to $100 \%$ by Park et al. ${ }^{[26]}$ This was probably due to the different types of psoriatic lesions being studied by them. Suprabasal mitoses were seen in $26 \%$ cases, comparable to Bai and Shrinivasan ${ }^{[29]}(36.67 \%)$.

Trozak Grading: No studies were found for the direct comparison of results obtained in our study. There was no particular trend seen according to age or sex of the patients or significant difference in early onset or late onset psoriasis. This highlights the variable manifestation of psoriasis at histological level.

\section{Conclusion}

Our study revealed that the characterstic features of Psoriasis are present in variable proportions. Nonspecific features like acanthosis and hyperkeratosis are seen universally and represent proliferative process while the specific features i.e. Micromunro's abscess and spongiform pustules of Kogoj are found in minority of cases and are signs of underlying inflammatory process. The other evidences of inflammation like presence of epidermal and dermal neutrophils and dermal mononuclear infiltration, especially perivascular, are seen more commonly. Parakeratosis and hypogranulosis is seen in majority of the cases, having uniform or more commonly focal distribution. Regular elongation of rete ridges with club shaped rete pegs and suprapapillary thinning are also prominent features. Early and Late onset psoriasis show differences in inflammatory features. The Trozak system is a simple and objective method to grade Psoriasis histologically which can be of significance to prescribe treatment or to assess the therapeutic efficacy.

\section{Acknowledgements}

We are thankful to faculty and technical staff of Pathology department and faculty of Dermatology department, GMC, Kota

\section{Reference}

1. Fry L. Psoriasis. Br J Dermatol 1988;119:445-61

2. World Health Organization. Global report on psoriasis. 2016. WHO Library Cataloguing-in-Publication Data. 2016.

3. Gibbs SA. Skin disease and socioeconomic conditions in rural Africa: Tanzania. International journal of dermatology. 1996 Sep;35(9):633-9.

4. Danielsen K, Olsen AO, Wilsgaard T, Furberg AS. Is the prevalence of psoriasis increasing? A 30-year follow-up of a population-based cohort. British Journal of Dermatology. 2013 Jun;168(6):1303-10.

5. Institute for Health Metrics and Evaluation (IHME). Global Burden of Disease Study 2010: Results by Cause 1990 2010. Seattle: IHME; 2012.
6. Boehncke W-H, Schön MP. Psoriasis. Lancet. 2015;386(9997):983-94.

7. Theodorakopoulou E. An investigation of late onset psoriasis (Doctoral dissertation, The University of Manchester (United Kingdom)). 2014.

8. Dubertret L, Mrowietz U, Ranki A, Van De Kerkhof PC, Chimenti S, Lotti T, Schäfer G, EUROPSO patient survey. European patient perspectives on the impact of psoriasis: the EUROPSO patient membership survey. British Journal of Dermatology. 2006 Oct;155(4):729-36.

9. Pandit GA, Narayankar SL. Significance of clinicopathological correlation in psoriasis. Medical Journal of Dr. DY Patil University. 2015 Jul 1;8(4):481.

10. Baker H, Ryan TJ. Generalized pustular psoriasis. British Journal of Dermatology. 1968 Dec 1;80(12):771-93

11. Ferrándiz C, Pujol RM, García-Patos V, Bordas X, Smandía JA. Psoriasis of early and late onset: a clinical and epidemiologic study from Spain. Journal of the American Academy of Dermatology. 2002 Jun 1;46(6):867-73.

12. Tuzun B. The Differential Diagnosis of Psoriasis Vulgaris. Pigmentary Disorders. 2016;3(245):2376-0427.

13. Krengel S, Schaumburg-Lever GM, Geilen CC, Orfanos CE. Histopathology and electron microscopy of psoriasis. BASIC AND CLINICAL DERMATOLOGY. 1998;16:409-20.

14. Mendonca SM, Devaraju S. Suprabasal mitotic index: A cell kinetic aid in psoriasis diagnosis. Indian Journal of Dermatopathology and Diagnostic Dermatology. 2017 Jan $1 ; 4(1): 2$.

15. De Rosa G, Mignogna C. The histopathology of psoriasis. Reumatismo. 2007;59(1s):46-8.

16. Raghuveer C, Shivanand DR, Rajashekar N. A Clinicohistopathological Study of Psoriasis. International Journal of Scientific Study. 2015 Oct 1;3(7):176-9.

17. Trozak DJ. Histologic grading system for psoriasis vulgaris. International journal of dermatology. 1994 May;33(5):380-1.

18. Icen M, Crowson CS, McEvoy MT, Dann FJ, Gabriel SE, Kremers HM. Trends in incidence of adult-onset psoriasis over three decades: a population-based study. Journal of the American Academy of Dermatology. 2009 Mar 1;60(3):394-401.

19. Moorchung N, Khullar JS, Mani NS, Chatterjee M, Vasudevan B, Tripathi T. A study of various histopathological features and their relevance in pathogenesis of psoriasis. Indian journal of dermatology. $2013 \mathrm{Jul} ; 58(4): 294$.

20. Henseler T, Christophers E. Psoriasis of early and late onset: characterization of two types of psoriasis vulgaris. Journal of the American Academy of Dermatology. 1985 Sep $1 ; 13(3): 450-6$

21. Ejaz A, Raza N, Iftikhar N, Iftikhar A, Farooq $M$. Presentation of early onset psoriasis in comparison with 
late onset psoriasis: A clinical study from Pakistan. Indian Journal of Dermatology, Venereology, and Leprology. 2009 Jan 1;75(1):36.

22. Bedi TR. Clinical profile of psoriasis in North India. Indian Journal of Dermatology, Venereology, and Leprology. 1995 Jul 1;61(4):202.

23. Kaur I, Kumar B, Sharma KV, Kaur S. Epidemiology of Psoriasis in a Clinic From North India. Indian journal of dermatology, venereology and leprology. 1986;52(4):20812.

24. Puri N, Mahajan BB. A study of clinical and biochemical correlation in patients of psoriasis in acute exacerbation. Journal of Pakistan Association of Dermatology. 2016 Dec 2;24(3):236-40.

25. Aydin O, Engin B, Oğuz O, İlvan Ş, Demirkesen C. Nonpustular palmoplantar psoriasis: is histologic differentiation from eczematous dermatitis possible?. Journal of cutaneous pathology. 2008 Feb;35(2):169-73.

26. Park JH, Park YJ, Kim SK, Kwon JE, Kang HY, Lee ES, Choi JH, Kim YC. Histopathological differential diagnosis of psoriasis and seborrheic dermatitis of the scalp. Annals of dermatology. 2016 Aug 1;28(4):427-32.

27. Kim BY, Choi JW, Kim BR, Youn SW. Histopathological findings are associated with the clinical types of psoriasis but not with the corresponding lesional psoriasis severity index. Annals of dermatology. 2015 Feb 1;27(1):26-31.

28. Lundin Å, Fredens K, Michaelsson G, Venge P. The eosinophil granulocyte in psoriasis. British Journal of Dermatology. 1990 Feb 1;122(2):181-93.

29. Bai S, Sowmya S. Histopathologic diagnostic parameters of psoriasis; a clinicopathological study. Int J Res Med Sci 2016;4:1915-20.

*Corresponding author:

Ila Sharma, B-39 Vallabh Nagar, Kota (Rajasthan) India

Phone: +91 8741916646

Email: drilasharma@live.com

Financial or other Competing Interests: None. 